\title{
ARITHMETIC STRUCTURE AND LACUNARY FOURIER SERIES
}

\author{
GEORGE BENKE ${ }^{1}$
}

\begin{abstract}
We prove a theorem concerning the arithmetic structure of $\Lambda(p)$ sets. This generalizes a result of Rudin and yields a new characterization of Sidon sets for certain Abelian groups.
\end{abstract}

Let $G$ be a compact Abelian group with dual group $\Gamma$. A subset $E \subset \Gamma$ is called a $\Lambda(p)$ set $(2<p<\infty)$ if for every trigonometric polynomial $f=\sum_{1}^{n} a_{k} \gamma_{k}\left(\gamma_{1}, \cdots, \gamma_{n} \in E\right)$ an inequality $\|f\|_{p} \leqq B\|f\|_{2}$ holds, where the constant $B$ depends only on $E$ and $p$. Theorems 1 and 2 of this paper give estimates on the number of elements of $E$ which can lie in a given multidimensional arithmetic progression in $\Gamma$. Theorem 2 is a generalization of Rudin's result [5, Theorem 3.5]. When a set $E$ is $\Lambda(p)$ for all $2<p<\infty$ and the constant $B$ may be taken as $C p^{1 / 2}$ where $C$ depends only on $E$ then it is called a $\Lambda$ set. A subset $E \subset \Gamma$ is said to be a Sidon set if every bounded function on $E$ can be extended to be a Fourier-Stieltjes transform. It is known that every Sidon set is a $\Lambda$ set, but the converse is unknown [4, p. 128]. As a corollary to Theorem 2 we find that $\Lambda$ sets are also Sidon sets in groups $\Gamma$ where each element has the same prime order.

Notation. Suppose $s$ and $N$ are positive integers, $1 \leqq r<\infty, b_{1}, \cdots$, $b_{k} \in \Gamma$ have finite order, $b_{k+1}, \cdots, b_{s} \in \Gamma$ have infinite order and $b_{0} \in \Gamma$ has arbitrary order. Set

$$
A_{r}(N, s, \boldsymbol{b})=\left\{\boldsymbol{n} \cdot \boldsymbol{b}+\left.b_{0}\left|\sum_{k+1}^{s}\right| n_{j}\right|^{r} \leqq N^{r}\right\}
$$

where $\boldsymbol{n} \cdot \boldsymbol{b}=n_{1} b_{1}+\cdots+n_{s} b_{s}$. Denote by $\hat{f}$ the Fourier transform of $f \in L^{1}(G)$, by $\langle x, \gamma\rangle$ the action of the character $\gamma$ on $x \in G$, and by $|F|$ the cardinality of a finite set $F$.

THEOREM 1. Let $b_{1}, \cdots, b_{k} \in \Gamma$ have finite orders $\beta_{1}, \cdots, \beta_{k}$, and let $b_{k+1}, \cdots, b_{s} \in \Gamma$ have infinite order. Let $E \subset \Gamma$ be $a \Lambda(p)$ set with $B$ a

Received by the editors September 24, 1971 and, in revised form, December 8, 1971. AMS 1970 subject classifications. Primary 42A44, 43A70.

Key words and phrases. Lacunary Fourier series, Sidon sets, $\Lambda(p)$ sets.

1 This work constitutes part of the author's doctoral dissertation at the University of Maryland under the direction of Professor J. J. Benedetto.

(C) American Mathematical Society 1972 
constant as in the definition of $\Lambda(p)$ set. If $A_{2}(N, s, b)=A_{2}$ is as defined above and $b_{k+1}, \cdots, b_{s}$ are independent in $\Gamma$ then

$$
\left|A_{2} \cap E\right| \leqq B^{2} e^{2}\left(\beta_{1} \cdots \beta_{k}\right)^{2 / p}\left(1+N(\pi / 2)^{1 / 2}\right)^{2(s-k) / p} .
$$

Proof. Since translates of $\Lambda(p)$ sets are $\Lambda(p)$ sets with the same constant of definition, we may take $b_{0}=0$. Define

$$
\begin{array}{ll}
g_{b_{j}}(x)=\sum_{n=0}^{\beta_{j}-1}\left\langle x, n b_{j}\right\rangle & \text { for } j=1, \cdots, k, \\
g_{b_{j}}(x)=\sum_{n \in Z} \exp \left(-n^{2} / N^{2}\right)\left\langle x, n b_{j}\right\rangle & \text { for } j=k+1, \cdots, s .
\end{array}
$$

It is easy to check that $g_{b_{j}}(x) \geqq 0$ for all $x \in G$.

Let $\Gamma^{\prime}=Z\left(\beta_{1}\right) \oplus \cdots \oplus Z\left(\beta_{k}\right) \oplus Z^{s-k}$, then

$$
g(x)=\sum_{n \in \Gamma^{\prime}} \exp \left(-\left\|n^{*} / N\right\|_{2}^{2}\right)\langle x, \boldsymbol{n} \cdot \boldsymbol{b}\rangle
$$

where $n^{*}=\left(0, \cdots, 0, n_{k+1}, \cdots, n_{s}\right)$. The map $\phi(n)=n \cdot b$ is a homomorphism of $\Gamma^{\prime}$ into $\Gamma$. If $H_{\gamma}=\phi^{-1}(\gamma)$ then

$$
\hat{g}(\gamma)=\sum_{n \in H_{\gamma}} \exp \left(-\left\|n^{*} / N\right\|_{2}^{2}\right)
$$

when $\gamma \in \operatorname{Im} \phi$ and $\hat{g}(\gamma)=0$ otherwise.

We will show that

$$
\hat{g}(0) \leqq e \hat{g}(\gamma) \text { for all } \gamma \in A_{2}(N, s, b) .
$$

Define an equivalence relation on $H_{0}$ by $h_{1}$ is related to $h_{2}$ if $h_{1}= \pm h_{2}$. Denote the quotient space by $\mathscr{H}$ and the class containing $h$ by [h]. Let $\gamma \in \operatorname{Im} \phi$ be fixed and take $n_{\gamma} \in H_{\gamma}$, then since $H_{\gamma}=n_{\gamma}+H_{0}$ we have

$$
\begin{aligned}
\hat{g}(\gamma) & =\sum_{\boldsymbol{m} \in \boldsymbol{H}_{0}} \exp \left(-\left\|\left(\boldsymbol{m}^{*}+\boldsymbol{n}_{\gamma}^{*}\right) / N\right\|_{2}^{2}\right) \\
& =\sum_{[\boldsymbol{m}] \in \mathscr{H}} \sum_{\boldsymbol{m} \in[\boldsymbol{m}]} \exp \left(-\left\|\boldsymbol{m}^{*} / N\right\|_{2}^{2}-\left\|\boldsymbol{n}_{\gamma}^{*} / N\right\|_{2}^{2}-2 \boldsymbol{m}^{*} \cdot \boldsymbol{n}^{*} / N^{2}\right) \\
& =\exp \left(-\left\|\boldsymbol{n}_{\gamma}^{*} / N\right\|_{2}^{2}\right)\left(1+2 \sum_{[\boldsymbol{m}] \in \mathscr{H}-[0]} \exp \left(-\left\|\boldsymbol{m}^{*} / N\right\|_{2}^{2}\right) \cosh \left(2 \boldsymbol{m}^{*} \cdot \boldsymbol{n}_{\gamma}^{*} / N^{2}\right)\right) .
\end{aligned}
$$

Hence

$$
\begin{aligned}
& \hat{g}(0)=1+2 \sum_{[m] \in \mathscr{H}-[0]} \exp \left(-\left\|\boldsymbol{m}^{*} / N\right\|_{2}^{2}\right) \\
& \hat{g}(\gamma) \geqq e^{-1}\left(1+2 \sum_{[m] \in \mathscr{H}-[0]} \exp \left(-\left\|\boldsymbol{m}^{*} / N\right\|_{2}^{2}\right)\right), \text { for }\left\|\boldsymbol{n}_{\gamma}^{*}\right\|_{2} \leqq N .
\end{aligned}
$$

Therefore (1) follows. 
Let $f$ be the trigonometric polynomial such that $\hat{f}(\gamma)=1$ for all $\gamma \in$ $E \cap A_{2}$ and $=0$ otherwise. Let $\alpha=\left|E \cap A_{2}\right|$ and $\delta=\min \left\{\hat{g}(\gamma) \mid \gamma \in A_{2}\right\}$. Then

$$
\begin{aligned}
\alpha & =\sum_{\gamma \in \Gamma} \hat{f}(\gamma) \\
& \leqq \sum_{\gamma \in \Gamma} \hat{f}(\gamma) \hat{g}(\gamma) / \delta=(1 / \delta) \int_{G} f(x) g(x) d x \\
& \leqq(1 / \delta)\|f\| g \|_{a D}
\end{aligned}
$$

where $1 / p+1 / q=1$. By the logarithmic convexity of the \|\|$_{q}$ norms and the fact that $\|g\|_{1}=\hat{g}(0)$, we have

$$
\|g\|_{q} \leqq\|g\|_{1}^{(2-q) / q}\|g\|_{2}^{(2 q-2) / q}=\hat{g}(0)\left(\|g\|_{2} /\|g\|_{1}\right)^{2 / p} .
$$

Therefore $\alpha \leqq\|f\|_{p}(\hat{g}(0) / \delta)\left(\|g\|_{2} /\|g\|_{1}\right)^{2 / p}$. It follows from the definition of $\Lambda(p)$ set and the estimate (1) that $\alpha \leqq B\|f\|_{2} e\left(\|g\|_{2} /\|g\|_{1}\right)^{2 / p}$ and since $\|f\|_{2}=\|\hat{f}\|_{2}=\alpha^{1 / 2}$,

$$
\alpha \leqq B^{2} e^{2}\left(\|g\|_{2} /\|g\|_{1}\right)^{4 / p} .
$$

It remains to estimate $\|g\|_{2} /\|g\|_{1}$. Generally

$$
\frac{\|g\|_{2}}{\|g\|_{1}}=\frac{\left(\sum_{\operatorname{cosets} H_{\gamma}}\left(\sum_{m \in H_{\gamma}} \exp \left(-\left\|m^{*} / N\right\|_{2}^{2}\right)\right)^{2}\right)^{1 / 2}}{\sum_{m \in H_{0}} \exp \left(-\left\|m^{*} / N\right\|_{2}^{2}\right)} .
$$

When $b_{k+1}, \cdots, b_{k}$ form an independent subset of $\Gamma$ it is easy to check that $H_{0}=0$. Therefore

$$
\|g\|_{2} /\|g\|_{1}=\left(\sum_{n \in \Gamma^{\prime}} \exp \left(-2\left\|m^{*} / N\right\|_{2}^{2}\right)\right)^{1 / 2}=\left(\left(\beta_{1} \cdots \beta_{k}\right)\left(1+(\pi / 2)^{1 / 2} N\right)^{s-k}\right)^{1 / 2}
$$

and we have the conclusion.

REMARK. In certain special cases like $s=2$ and $\Gamma=Z$, even when $H_{0} \neq 0$ explicit computations show that $\|g\|_{2} /\|g\|_{1}=O\left(N^{s / 2}\right)$. However, we are unable to establish this for the general case.

THEOREM 2. Let $b_{1}, \cdots, b_{k} \in \Gamma$ have finite orders $\beta_{1}, \cdots, \beta_{k}$ and $b_{k+1}, \cdots, b_{s} \in \Gamma$ have infinite order. Let $E \subset \Gamma$ be $a \Lambda(p)$ set $B$ as in the definition of $\Lambda(p)$ set. If $1 \leqq r<\infty$ then

$$
\left|A_{r}(N, s, b) \cap E\right| \leqq B^{2} e^{2}\left(\beta_{1} \cdots \beta_{k}\right)^{2 / p}\left(1+N^{r}\right)^{2(s-k) / p} .
$$

Proof. Again without loss of generality we take $b_{0}=0$. Consider first the case $r=1$. Define $g_{b_{1}}, \cdots, g_{b_{k}}$ as in the proof of Theorem 1, and for $j=k+1, \cdots, s$ define

$$
g_{b_{j}}(x)=\sum_{n \in Z} \exp \left(-\|n / N\|_{1}\right)\left\langle x, n b_{j}\right\rangle .
$$


Let $g(x)=\prod_{j=1}^{s} g_{b_{j}}(x)$ then $\hat{g}(\gamma) / \hat{g}(0)>e$ for all $\gamma \in A_{1}$ and, therefore, $\left|A_{1} \cap E\right| \leqq B^{2} e^{2}\left(\|g\|_{2} /\|g\|_{1}\right)^{4 / p}$. Let $n_{\gamma}$ be any one element in the coset $H_{\gamma}$; then as before

$$
\begin{aligned}
\|g\|_{2} & =\left(\sum_{\operatorname{cosets} H_{\gamma}}\left(\sum_{m \in H_{0}} \exp \left(-\left\|\left(\boldsymbol{m}^{*}+\boldsymbol{n}_{\gamma}^{*}\right) / N\right\|_{1}\right)\right)^{2}\right)^{1 / 2} \\
& \leqq\left(\sum_{\operatorname{cosets} H_{\gamma}} \exp \left(-2\left\|\boldsymbol{n}_{\gamma}^{*} / N\right\|_{1}\right)\left(\sum_{\boldsymbol{m} \in H_{0}} \exp \left(-\left\|\boldsymbol{m}^{*} / N\right\|_{1}\right)\right)^{2}\right)^{1 / 2} .
\end{aligned}
$$

Since $\|g\|_{1}=\hat{g}(0)=\sum_{m \in H_{0}} \exp \left(-\left\|m^{*} / N\right\|_{1}\right)$ we have

$$
\begin{aligned}
\|g\|_{2} /\|g\|_{1} & \leqq\left(\sum_{\operatorname{cosets} H_{\gamma}} \exp \left(-2\left\|\boldsymbol{n}_{\gamma}^{*} / N\right\|_{1}\right)\right)^{1 / 2} \\
& \leqq\left(\sum_{n \in \Gamma^{\prime}} \exp \left(-2\left\|n^{*} / N\right\|_{1}\right)\right)^{1 / 2}\left(\left(\beta_{1} \cdots \beta_{k}\right)(1+N)^{s-k}\right)^{1 / 2},
\end{aligned}
$$

and the conclusion follows for $r=1$. Now suppose $1<r<\infty$. The trivial inequality $\sum_{j=k+1}^{s}\left|n_{j}\right| \leqq \sum_{j=k+1}^{s}\left|n_{j}\right|^{r}$ shows that $A_{r}(N, s, b) \subset A_{1}\left(N^{r}, s, b\right)$ and the conclusion follows for the general case.

Corollary. Suppose $E \subset \Gamma$ is a $\Lambda$ set, then

$$
\left|A_{r} \cap E\right| \leqq 2 C^{2} e^{3} \log \left(\left(\beta_{1} \cdots \beta_{k}\right)\left(1+N^{r}\right)^{s-k}\right)
$$

where $C$ is the constant in the definition of $\Lambda$ set.

Proof. In Theorem 2 set $p=2 \log \left(\left(\beta_{1} \cdots \beta_{k}\right)\left(1+N^{r}\right)^{s-k}\right)$.

Kahane [2, p. 58] has already proven this result for the case where $E$ is a Sidon set, and it is unknown if this condition is sufficient to make a set Sidon. The next result shows that in certain cases it is and therefore gives equality of Sidon and $\Lambda$ sets (cf. M. P. Malliavin-Brameret and P. Malliavin [3]).

COROllary. Let $E \subset \Gamma$ and suppose that each $\gamma \in \Gamma$ has prime order $\beta$. Then the following are equivalent.

(i) $\left|A_{1} \cap E\right| \leqq K s$ ( $K$ depends only on $E$ ).

(ii) $E$ is a Sidon set.

(iii) $E$ is a $\Lambda$ set.

Proof. We know that (ii) $\Rightarrow$ (iii) $\Rightarrow$ (i). Therefore, we show (i) $\Rightarrow$ (ii). The following result is due to Horn [1].

Let $V$ be a vector space over some field and $E \subset V$. Given a natural number $K$, the following two statements are equivalent.

(a) $E$ is the union of $K$ linearly independent sets.

(b) $|B| \leqq K \operatorname{rank}(B)$ for all finite subsets $B \subset E$, where $\operatorname{rank}(B)$ is defined as the number of elements in a maximal linearly independent subset of $B$. 
$\Gamma$ is a vector space over $Z(\beta)$, and (i) implies that statement (b) of Horn's theorem is satisfied. Thus $E$ is the union of $K$ linearly independent sets and is, therefore, a Sidon set [4, p. 124].

\section{REFERENCES}

1. A. Horn, A characterization of unions of linearly independent sets, J. London Math. Soc. 30 (1955), 494-496. MR 17, 135.

2. J. P. Kahane, Some random series of functions, Heath, Lexington, Mass., 1968. MR 40 \#8095.

3. M. P. Malliavin-Brameret and P. Malliavin, Caractérisation arithmétique d'une classe d'ensembles de Helson, C. R. Acad. Sci. Paris 264 (1967), 192-193.

4. W. Rudin, Fourier analysis on groups, Interscience Tracts in Pure and Appl. Math., no. 12, Interscience, New York, 1962. MR 27 \#2808.

5. - Trigonometric series with gaps, J. Math. Mech. 9 (1960), 203-227. MR 22 \#6972.

Department of Mathematics, Georgetown University, Washington, D.C. 20007 